\title{
Evaluating Applied Flight-deck Interval Management using Monte Carlo Simulations on the K-Supercomputer*
}

\author{
Timo RIEDEL, ${ }^{1), 2) \vdots}$ Masaki TAKAHASHI, ${ }^{1)}$ Tomoaki TATSUKAWA, ${ }^{3)}$ and Eri ITOH ${ }^{2)}$ \\ ${ }^{1)}$ Graduate School of Science and Technology, Keio University, Yokohama, Kanagawa 223-8522, Japan \\ ${ }^{2)}$ Air Traffic Management Department, Electronic Navigation Research Institute, Chofu, Tokyo 182-0012, Japan \\ ${ }^{3)}$ Department of Information and Computer Technology, Tokyo University of Science, Tokyo 125-8585, Japan
}

\begin{abstract}
Increasing air traffic and airport congestion calls for solutions to improve traffic throughput. In this paper, we evaluate one solution, Flight-deck Interval Management and its underlying logic, particularly in terms of its utility. An environment to simulate traffic engaged in interval management on the most common arrival routes toward Tokyo International Airport was set up and run as a large-scale Monte Carlo simulation on the K-Supercomputer. The results show that under low wind conditions in particular, spacing goal times with a standard deviation of less than $5 \mathrm{~s}$ can be achieved and a speed tolerance mode can reduce the number of speed commands without compromising performance.
\end{abstract}

Key Words: Airborne Spacing Interval Management, Arrival Management, Tokyo International Airport, Control Logic, Operational Feasibility

\section{Introduction}

Flight-deck Interval Management (FIM) as part of Airborne Spacing Interval Management has been proposed by the ICAO Global Air Navigation Plan ${ }^{1)}$ as a solution to improve air traffic efficiency and runway utilization, and is being considered for implementation within Japan's Collaborative Actions for Renovation of Air Traffic Systems (CARATS) activities. ${ }^{2)}$

Previous studies have examined the application of FIM at Japanese airports, especially Tokyo International Airport $(\text { RJTT })^{3-6)}$; however, owing to the computation time required, they have been limited to selected scenarios, and thus far, no study has comprehensively analyzed the utility of FIM for the most common arrival routes.

Furthermore, a qualitative flight crew survey conducted during a flight test in February 2017 indicated that the system has not yet attained an operationally implementable level owing to issues related to workload and acceptance. ${ }^{7-9)}$ This was reflected by negative feedback comments such as "Too many speed changes," "Large decelerations" (speed steps well over $40 \mathrm{kt}$ ), and "inefficient IM speed reversals" (speed commands negating the effect of a previous command). Previous studies have primarily focused on the goal time observation or the string stability of multiple aircraft engaged in FIM, ${ }^{10)}$ while usability factors have rarely been considered. Hence, it remains difficult to identify the above-mentioned phenomena and their impacts.

In this study, we run large-scale Monte Carlo simulations on the RIKEN K-Supercomputer, ${ }^{11)}$ which enables us to overcome the computation time problem. Additionally, we add parameters related to items mentioned in the flight test's

(C) 2019 The Japan Society for Aeronautical and Space Sciences

*Received 4 November 2018; final revision received 20 April 2019; accepted for publication 13 June 2019.

‡Corresponding author, timo.riedel@keio.jp qualitative survey. By analyzing the distribution and deviation of these parameters, we can compare different logics and modes in terms of their utility and impact on spacing performance and speed commands, which enables us to propose changes to current FIM logics and identify future tasks before devoting human and financial resources to another Human-in-the-Loop (HITL) experiment.

The remainder of this paper is organized as follows. Section 2 introduces FIM, its underlying logic, and previous research results. Section 3 describes the simulation setup, initial conditions, and reasons for choosing the parameters. Section 4 presents the detailed simulation results. Section 5 discusses the results. Finally, Section 6 concludes the paper by stating some recommendations and exploring directions for future research.

\section{Flight-deck Interval Management}

\subsection{General concept}

FIM is a ground-equipment-independent airborne IM solution that transfers the spacing adjustment and observation tasks from the Air Traffic Controller (ATCO) to the flight crew. It is based on the principle of calculating the trajectories of the primary aircraft ("Ownship") and a pre-selected leading aircraft ("Traffic-To-Follow," TTF) and comparing their estimated times of arrival (ETAs) for a common waypoint (e.g., the Achieve-by-Point, ABP; see Fig. 1).

The difference between the Ownship and TTF ETAs plus the nominal spacing $(\Delta)$ equals the spacing error ${ }^{12)}$ :

$$
e(t)=\operatorname{ETA}_{\text {ownship }}(t)-\left(\operatorname{ETA}_{\mathrm{TTF}}(t)+\Delta\right) .
$$

FIM tries to eliminate this error. The necessary data for trajectory calculation is obtained from the flight management system (FMS) of the Ownship and ADS-B (out) signal of the TTF. 


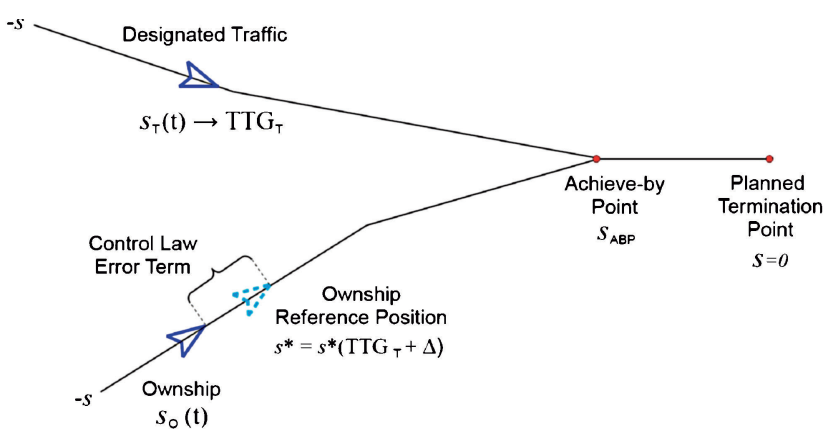

Fig. 1. Example of the time-based spacing goal, trajectory-based operation logic for traffic toward a common waypoint. ${ }^{12)}$

\subsection{Airborne spacing for terminal arrival routes (ASTAR)}

ASTAR is a control concept for FIM that was first introduced by NASA's Langley Research Center in 2002. Designed as a fully integrated, auto-pilot and auto-throttle connected system, ASTAR issues airspeed commands to minimize the spacing error. The system was redesigned as a retrofittable federated system as part of NASA's Air Traffic Demonstration 1 (ATD-1) Program, in which it is implemented as an application running on an Electronic Flight Bag (EFB). ${ }^{13-16)}$ The appropriate IM speed is recommended to pilots, who then set this speed on the auto-pilot control panel. ASTAR offers a trajectory-based operation (TBO) mode for merging traffic and a constant time delay (CTD) mode for traffic on the same path. The latest version, ASTAR13, was released in August 2015, and was evaluated during a flight test in February 2017 (see below). ASTAR works on a direct feed-forward principle; a controller chart can be found in Ref. 13). In this study, we focus on the TBO part of ASTAR.

\subsection{Flight test results}

NASA, together with Boeing, United Airlines, and Honeywell, conducted a 19-day flight test experiment for not only evaluating the spacing performance, but also surveying the flight crew with regard to their perception of operational feasibility and task workload. Although the spacing performance was satisfactory, operational issues that are barely identifiable outside an HITL environment were found in terms of the number, frequency, and timing of speed commands, their magnitude (step size), and overall energy management. ${ }^{7-9)}$

The parameters measured in our simulation (described in Section 3.11) were selected to quantify these findings.

\subsection{Previous simulations}

In our previous studies, an ASTAR10-based algorithm in an automated configuration was evaluated for its spacing performance on standard terminal arrival routes (STAR). ${ }^{6}$ This was compared with the performance of ASTAR13, including federated modes, on selected routes toward the terminal entry. ${ }^{5)}$ In the latter, the number and frequency of speed commands issued were investigated. The results showed that version 13 is slightly better than version 10 and supported some of the flight test comments. Further testing showed that although certain measures for reducing the number of speed commands (see Section 3.5) can be effective, they affect spacing performance.

\section{Simulation Setup}

\subsection{K-Supercomputer}

The RIKEN K-Supercomputer (K), manufactured by Fujitsu, was put into service in June 2011, and was the fastest supercomputer at that time. With over 80,000 nodes consisting of octa-core processors operating at $2.0 \mathrm{GHz}, \mathrm{K}$ reached its benchmark peak performance of 10.5 petaflops in November 2011. ${ }^{11)}$ As of June 2018, K was ranked 16th among the TOP500 supercomputer benchmark, and its successor "Post$\mathrm{K}$ " is scheduled to commence service in 2021. In our simulation, we used K's node network by simultaneously running the computation over 120 nodes, which enabled us to complete our calculations, which otherwise would have taken roughly 350 days on a single machine, in less than three days.

\subsection{SPICA}

Our simulation is based on the SPICA simulation environment, developed by the Electronic Navigation Research Institute (ENRI) in collaboration with NASA. ${ }^{3,4)}$ SPICA includes a route and trajectory generator, wind, pilot, autopilot, and a FIM speed control module. ${ }^{4)}$ Aircraft models are available for the B737-800, B767-300, B777-200, and B787-8. The route, wind and aircraft data are used as inputs with which SPICA can calculate the trajectory, including FIM commands, for up to 20 aircraft per scenario. Consecutive FIM operation is limited to four aircraft. Furthermore, a speed brake model is not available. Additional information on the trajectory calculation algorithm can be found in Refs. 14) and 15). Finally, the aircraft state log and FIM command summary are output by the simulator. An adapted $\mathrm{C}++$ version of SPICA is used on $\mathrm{K}$.

\subsection{Routing}

All routings were chosen in accordance with the preferred routings and STARs as per Aeronautical Information Circular NR014/18, effective as of April 26th, 2018. For our simulation, we chose scenarios based on the merging point of two aircraft. Thus, en-route scenarios starting from cruising altitude to one of the terminal entry points and arrival scenarios from the entry point to the appointed runway threshold were defined.

\subsubsection{Lateral path (en-route scenarios)}

The en-route scenarios start at approximately 200 NM toward RJTT and end at one of the waypoints ADDUM, STONE or KAIHO (see Fig. 2). The ADDUM entry is preferred for domestic traffic from the west of Japan (initial waypoint SHTLE), Kyushu (FLUTE), and Okinawa (SOPHY), as well as international traffic from Southeast Asia, Australia, and Hawaii.

The STONE entry is preferred for northern domestic traffic (ZAHAN) and international routes from Europe (via RNAV route Y301) and North America (ESCAL).

ADDUM and STONE serve as the main STAR entry points (see arrival scenarios), and traffic from both of the waypoints can merge afterward if assigned the same runway. 

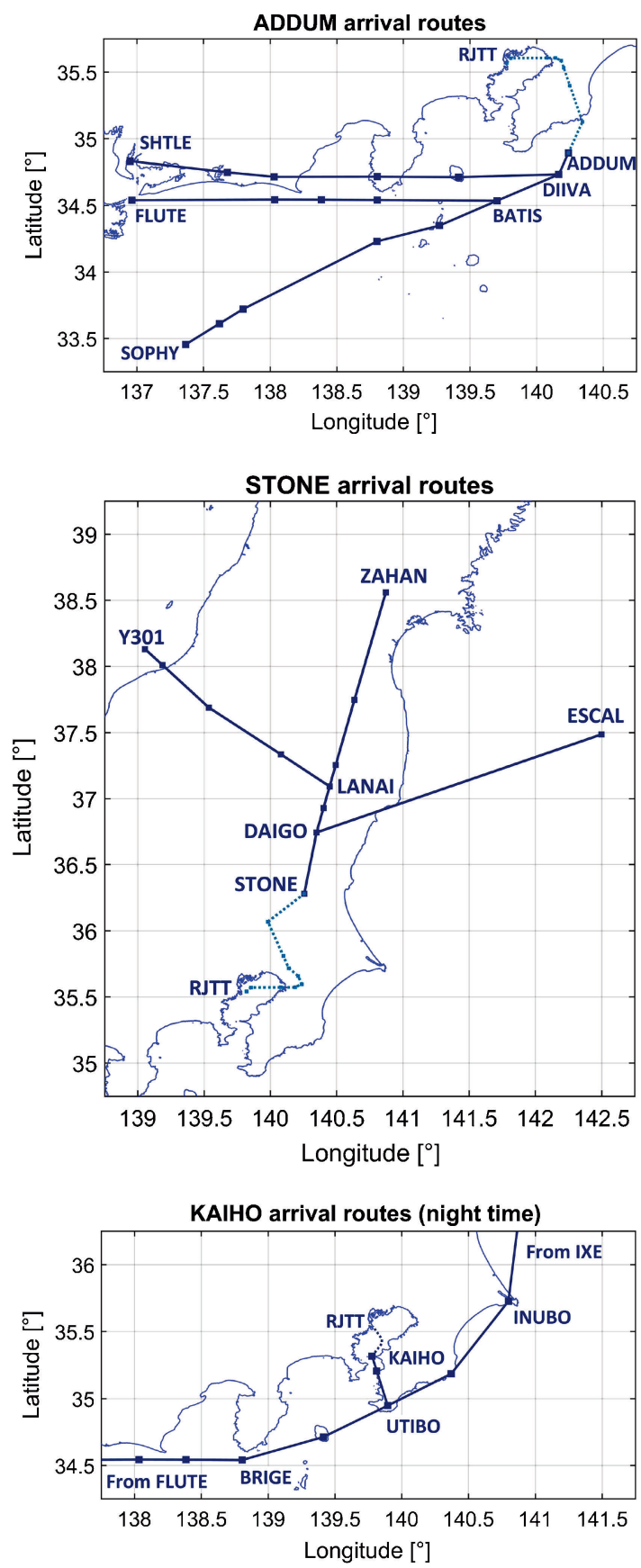

Fig. 2. Individual routings for the en-route scenarios to the terminal area entry points ADDUM, STONE, and KAIHO.

Table 1. En-route scenario identification.

\begin{tabular}{clcc}
\hline Case ID & Initial WP & Final WP & Distance [NM] \\
\hline \multirow{3}{*}{ AD1 $/ 2$} & SHTLE & ADDUM & 168.9 \\
& FLUTE & $10.000 \mathrm{ft} @ 230 \mathrm{kt}$ & 171.2 \\
& SOPHY & & 169.7 \\
\hline \multirow{2}{*}{$\mathrm{ST} 1 / 2$} & ZAHAN & STONE & 139.9 \\
& Y301 & 11.000 ft @250 kt & 144.7 \\
& ESCAL & & 139.7 \\
\hline \multirow{2}{*}{ KH1 $/ 2$} & FLUTE & KAIHO & 172.7 \\
& IXE & 4.000 ft @ 230 kt & 171.7 \\
\hline
\end{tabular}

Finally, the KAIHO entry represents a special scenario for night hours and low traffic cases. All of the traffic in this scenario, either from the west via FLUTE or the north via IXE, is merged at, or around, UTIBO and consecutively routed through Tokyo Bay to KAIHO. The specific paths are shown in Fig. 2 and the case identifiers are listed in Table 1.

\subsubsection{Lateral path (arrival scenarios)}

In the arrival scenarios, traffic is guided from ADDUM or STONE on the ARLON/CREAM arrival toward the 
RWY34L or 34R ILS (Z) approach (see Ref. 17) p. 35f) or on the BACON/DATUM arrival for a consecutive RWY22 or RWY23 LDA (W or Z) approach (see Ref. 17) p. 44f). Whereas traffic from ADDUM is usually guided toward RWY34L or RWY22 (see Fig. 3), traffic from STONE is guided toward RWY34R or RWY23. Under certain circumstances (runway closure, taxiing convenience, etc.), traffic can be routed to the opposite runway just before the Final Approach Fix. Figure 3 shows primary traffic as a solid line and secondary traffic as a dashed line, and Table 2 lists the abbreviated identifiers.

Traffic from KAIHO can be guided toward any runway. As the traffic was already merged, no connecting scenario was simulated.

\subsubsection{Vertical path}

All scenarios were simulated as Continuous Descent Operations (CDO) with Fixed Flight-path Angle (FPA) descents. ${ }^{18)}$ Previous studies have shown that fixed FPAs of up to $-2.7^{\circ}$ are manageable with regard to the pilot workload. ${ }^{19)}$ In this simulation, a shallower angle of $-2.2^{\circ}$ was utilized to allow for a greater margin during deceleration segments. Once the ILS glide slope has been captured, the standard angle of $-3.0^{\circ}$ is re-assumed.

The en-route scenarios uniformly start at an altitude of $38,000 \mathrm{ft}$ and end at the specified waypoint altitude of $11,000 \mathrm{ft}$ (STONE), $10,000 \mathrm{ft}$ (ADDUM), or $4,000 \mathrm{ft}$ (KAIHO). The arrival scenarios continue from this altitude to the runway threshold.

\subsection{Initial and final speeds}

For the en-route scenarios, all aircraft start with an initial speed of Mach 0.83. The final speed depends on the final waypoint: here, $250 \mathrm{kt}$ (STONE) and $230 \mathrm{kt}$ (ADDUM and KAIHO). Arrival scenarios start with the above-mentioned final speeds and continue toward the aircraft-specific final approach speed over the runway threshold.

\subsection{Speed modes}

With the change from an integrated system to a federated system, the following speed modes have been established.

\subsubsection{Automated}

This mode represents the originally intended fully integrated system. Speeds are set at intervals of $0.1 \mathrm{kt}$ and no interaction is required. This mode is evaluated for reference.

\subsubsection{Federated (Stepped)}

The federated mode requires pilots to manually insert all speed settings. Therefore, to maintain the number of commands at a low level, commands are issued at discretized intervals of $5 \mathrm{kt}$ (or Mach 0.01).

\subsubsection{End-speed}

An issue for the manual federated mode is shown in Fig. 4. During deceleration segments, pilots need to constantly monitor and change the speed input to "chase" the nominal profile, resulting in the "FED" profile shown in Fig. 4.

To prevent this behavior, the end-speed logic was introduced. At the beginning of deceleration, this logic sets the FIM speed command to the final waypoint-restricted speed (dashed line) instead of implementing a stepped decrease.

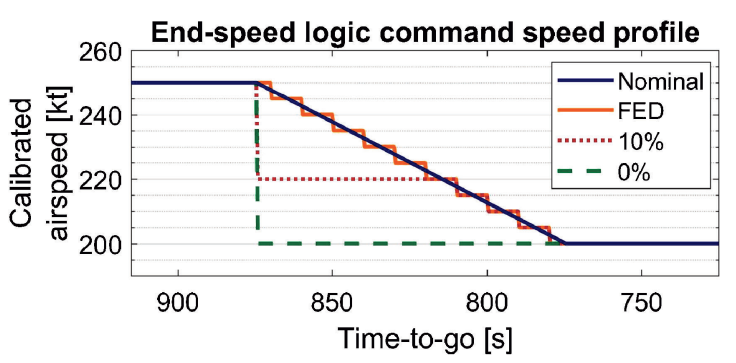

Fig. 4. End-speed logic command speeds.

The nominal and federated mode speeds are shown by solid lines, while the end-speed modes with tolerance are shown by dashed lines.

Table 3. Selected aircraft type probabilities.

\begin{tabular}{cccc}
\hline B737 & B767 & B777 & B787 \\
\hline $42 \%$ & $22 \%$ & $24 \%$ & $12 \%$ \\
\hline
\end{tabular}

Although this approach reduces the number of inputs, it also allows further deviation from the nominal profile, thereby affecting spacing performance. A trade-off between the number of commands and performance can be achieved by adding a tolerance to the restricted final speed (dotted line) and proceeding from this value as required. Here, we simulated tolerances of $0 \%, 5 \%, 10 \%$ (ASTAR10 speed tolerance), $15 \%$ (ASTAR13), and 20\% to achieve a suitable trade-off.

\subsection{Wind}

Two different wind conditions, i.e., a winter scenario with weather data from February 14, 2017, 12:00 UTC, and a summer scenario with weather data from August 8, 2017, 18:00 UTC, were considered. Over the main islands of Japan, the westerlies, generating winds aloft with speeds of $100 \mathrm{kt}$ or higher at FL300, usually prevail from October through June and move northward during the summer months. This difference is represented by the two above-mentioned scenarios. The en-route scenarios were simulated for both conditions and the arrival scenarios were simulated according to the prevailing wind conditions at the runway threshold (here, RWY34L/R for winter and RWY22/23 for summer).

\subsection{Aircraft types}

The aircraft type was randomly chosen on the basis of weighted probabilities. In our previous study, ${ }^{5)}$ we surveyed the distribution of the types of aircraft landing at RJTT. Aircraft models that were not covered were assigned to the closest category. The distribution is shown in Table 3.

\subsection{Initial spacing}

The initial start time for each aircraft in each scenario was determined such that two consecutive B737, not engaged in FIM, flying their nominal speed profile would arrive with a final spacing error of zero, regardless of their initial waypoint. As in previous simulations, ${ }^{5)}$ a random spacing error in the range of $-15 \mathrm{~s}$ to $+15 \mathrm{~s}$ was added initially, resulting in coupled spacing errors between $-30 \mathrm{~s}$ and $+30 \mathrm{~s}$. The overall distribution can be seen in Fig. 5.

\subsection{Initial sequence}

While the aircraft sequence (initial waypoints) for each scenario was fixed, it was ensured that each cross-combina- 


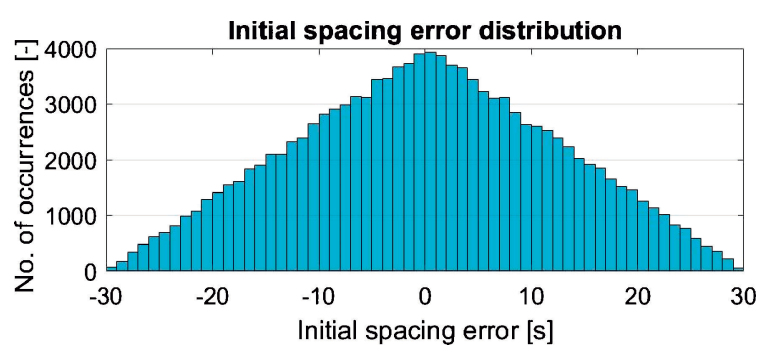

Fig. 5. Exemplary distribution of the initial spacing error for all cases.

tion (a total of 9 (4) possibilities for 3 (2) initial waypoints) was simulated at least once.

\subsection{Number of simulations}

In each run, 16 aircraft were simulated, among which 12 engaged in FIM operation. For each scenario (10 in total) and logic (6 in total), 10,000 runs were performed, resulting in 120,000 sets of data for each of the 60 conditions.

\subsection{Parameters evaluated}

In addition to the final spacing performance (i.e., mean, median, and standard deviation at the $\mathrm{ABP}$ ), we evaluated the total number of speed commands, number of speed commands issued in less than $60 \mathrm{~s}$, maximum speed step magnitude, and number of reversals.

\section{Simulation Results}

In this section, the results for each individual scenario are presented in three parts. In the first part, the final spacing error is shown using a histogram and boxplots. The histogram indicates the distribution of the final error for the unrestricted federated logic mode (i.e., without end-speed logic) in the foreground and the fully restricted $0 \%$ tolerance end-speed mode in the background. Each bar represents a range of $1 \mathrm{~s}$ and its height indicates the number of occurrences. For enroute (arrival) scenarios, the top of the histogram ends above $20,000(55,000)$ occurrences. Each route is shown in a different color for better readability.

The boxplots under the histogram show the error distribution by logic and tolerance for the end-speed mode. AUTO indicates the automated mode (given for reference) and FED indicates the unrestricted federated mode without end-speed logic. The percentages correspond to the endspeed logic tolerance.

The boxplots are shown from the $25 \%$ quartile to the $75 \%$ quartile, covering $50 \%$ of all the data points. The median value is indicated by the vertical bar within each box. The whiskers (outer markers) are set in accordance with the Tukey convention, extending the box by 1.5 times the inter-quartile range (IQR) and thus covering $99.3 \%$ of all the data points for normal distributed values. The outliers have been omitted for better visibility.

The second part includes two sets of boxplots. The upper set indicates the distribution of the total number of speed commands over the scenario and the lower set indicates the number of speed commands issued less than $60 \mathrm{~s}$ since the last FIM speed command.
The last part includes a table that lists the median, mean, and standard deviation of the final error, the median of the maximum speed change magnitude, and the range of the number of reversals from the $25 \%$ quartile to the $75 \%$ quartile, with the median in bold (if coinciding with a quartile value).

\subsection{En-route scenarios}

Figures 6 and 7, and Tables 4 and 5 show the results for the en-route scenario toward KAIHO. For the winter scenario, the box covering the final spacing error for the AUTO mode begins at $2.1 \mathrm{~s}$ ( $25 \%$ quartile), has its median at $4.0 \mathrm{~s}$, and ends at $6.4 \mathrm{~s}$ (75\% quartile). For the unrestricted FED mode and $0 \%$ tolerance end-speed mode, the corresponding values are $[2.5 \mathrm{~s}, 4.3 \mathrm{~s}$, and $6.6 \mathrm{~s}]$ and $[1.2 \mathrm{~s}, 4.0 \mathrm{~s}$, and $19.6 \mathrm{~s}]$, respectively. The median number of FIM speed commands ranges from 25 (FED) to 14 (0\%), of which 18 (8) are issued within $60 \mathrm{~s}$ of the previous command. In most cases, one or two reversals occur, with a slight reduction toward lower tolerance values. The summer scenario renders similar results, with slightly higher averages and deviations, i.e., [2.1s, $4.3 \mathrm{~s}$, and $6.9 \mathrm{~s}$ ] for AUTO, [2.7s, $4.6 \mathrm{~s}$, and $7.2 \mathrm{~s}$ ] for FED and $[0.9 \mathrm{~s}, 4.0 \mathrm{~s}$, and $23.5 \mathrm{~s}]$ for $0 \%$. However, in both the cases, the standard deviation remains under $5 \mathrm{~s}$ for tolerances of $10 \%$ or more.

Figures 8 and 9, and Tables 6 and 7 show the results for the ADDUM entry. For the winter scenario, the spacing error quantiles can be found at $[-4.5 \mathrm{~s},-1.4 \mathrm{~s}$, and $1.3 \mathrm{~s}]$ for AUTO, [-5.4 s, $-2.3 \mathrm{~s}$, and $0.5 \mathrm{~s}]$ for FED, and [-5.2 s, $1.3 \mathrm{~s}$, and $10.1 \mathrm{~s}$ ] for the $0 \%$ tolerance mode. The median number of speed commands ranges from 25 to 15 . In more than $50 \%$ of the simulations, 1 to 3 reversals occur. The results for the summer scenario show a small improvement in spacing performance: $[-2.8 \mathrm{~s},-0.3 \mathrm{~s}$, and $1.7 \mathrm{~s}$ ] for AUTO, $[-3.4 \mathrm{~s},-0.7 \mathrm{~s}$, and $1.9 \mathrm{~s}]$ for FED, and $[-2.3 \mathrm{~s}$, $3.8 \mathrm{~s}$, and $13.6 \mathrm{~s}$ ] for $0 \%$; however, a significant reduction in the number of FIM commands, with medians ranging from 18 to 7 , and reversals, with a uniform median of 1 , can be observed. The comparison highlights the difference in wind conditions between the summer and winter scenarios. This result and the impact of wind effects on the spacing performance are discussed in detail in Section 5.3. In both scenarios, the median values for the final spacing error remain close to the $0 \mathrm{~s}$ mark, while the standard deviation remains below the $5 \mathrm{~s}$ target only for the summer scenario in the AUTO, FED, and $20 \%$ modes.

The STONE entry is shown in Figs. 10 and 11, and Tables 8 and 9 . In the histogram for the winter scenario, a notable trail toward the positive end (delayed arrival) can be seen, which causes a larger spread between the mean and median values. Here, the quantiles can be found at $[-2.4 \mathrm{~s}, 0.7 \mathrm{~s}$, and $4.4 \mathrm{~s}]$ for AUTO, [-2.4s, 0.9 s, and $4.8 \mathrm{~s}]$ for FED, and [ $-0.8 \mathrm{~s}, 3.6 \mathrm{~s}$, and $14.5 \mathrm{~s}$ ] for $0 \%$. The median number of speed commands ranges from 27 to 16 . In the histogram for the summer scenario, an extended tail is not present. Instead of one distinct peak, two peaks or a flattened plateau can be found. The characteristic values lie at $[-0.7 \mathrm{~s}, 1.9 \mathrm{~s}$, and $5.1 \mathrm{~s}]$ for AUTO, $[-0.6 \mathrm{~s}, 2.7 \mathrm{~s}$, and 

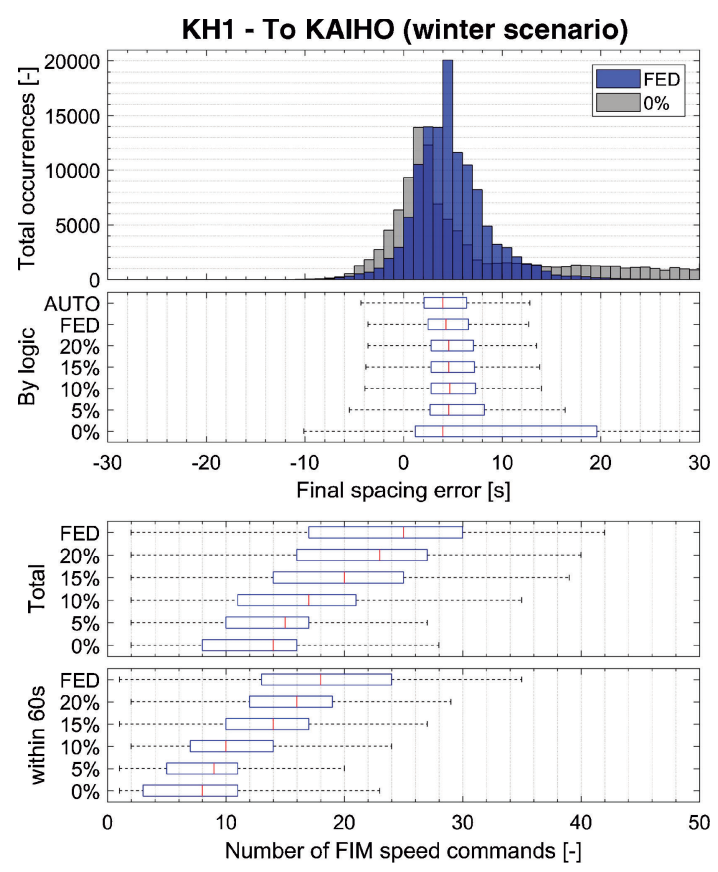

Fig. 6. Results for the en-route scenario to KAIHO (winter data).

Table 4. Accompanying table for KAIHO (winter data).

\begin{tabular}{lccccccc}
\hline \multicolumn{1}{c}{ Logic } & AUTO & FED & $20 \%$ & $15 \%$ & $10 \%$ & $5 \%$ & $0 \%$ \\
\hline Median error [s] & 4.0 & 4.3 & 4.6 & 4.6 & 4.7 & 4.6 & 4.0 \\
Avg. error [s] & 4.52 & 4.72 & 5.20 & 5.25 & 5.43 & 7.05 & 11.51 \\
Std. dev. [s] & 3.50 & 3.69 & 3.84 & 3.90 & 4.16 & 7.55 & 15.13 \\
Mag. (median) [kt] & - & 16 & 16 & 25 & 39 & 49 & 59 \\
Rev. (quartiles) [-] & - & $\mathbf{2 - 1}$ & $\mathbf{2 - 1}$ & $\mathbf{2 - 1}$ & $\mathbf{2 - 1}$ & $\mathbf{2 - 1}$ & $\mathbf{2 - 1}$ \\
\hline
\end{tabular}

$6.0 \mathrm{~s}$ ] for FED, and [3.3 s, $7.2 \mathrm{~s}$, and $11.4 \mathrm{~s}$ ] for $0 \%$, with the median number of speed commands lying in a similar range of 27 to 15 . As with the ADDUM scenarios, the standard deviation is found to be higher than $5 \mathrm{~s}$ except for the AUTO and FED modes in the summer scenario. The median number of reversals is uniformly found at 1 . These results can be attributed to the direction of the routes that merge toward STONE and the prevalent wind conditions. For further details, refer to Section 5.3.

\subsection{Arrival scenarios}

Figures 12 and 13, and Tables 10 and 11 show the results toward RWY34L and 34R. The final spacing error quantiles for RWY34L can be found at [2.4 s, $3.8 \mathrm{~s}$, and $4.7 \mathrm{~s}]$ for AUTO, [2.0 s, $3.0 \mathrm{~s}$, and $4.0 \mathrm{~s}]$ for FED, and [6.7 s, $9.2 \mathrm{~s}$, and $15.6 \mathrm{~s}$ ] for $0 \%$, with the median number of speed commands ranging from 14 to 6 . RWY34R yields comparable results, i.e., [ $2.9 \mathrm{~s}, 3.7 \mathrm{~s}$, and $5.1 \mathrm{~s}$ ] for AUTO, [2.3 s, $3.0 \mathrm{~s}$, and $4.1 \mathrm{~s}$ ] for FED, and [7.2 s, $9.9 \mathrm{~s}$, and $15.7 \mathrm{~s}$ ] for $0 \%$, with the median number of speed commands ranging from 14 to 7 . The standard deviation remains under $5 \mathrm{~s}$ for all of the modes with $5 \%$ or more tolerance and under $3 \mathrm{~s}$ for the modes with $15 \%$ or more tolerance. The median number of reversals is uniformly one with the exception of the FED mode in winter (2).

Figures 14 and 15, and Tables 12 and 13 show the results toward RWY22 and RWY23, respectively. The final spacing
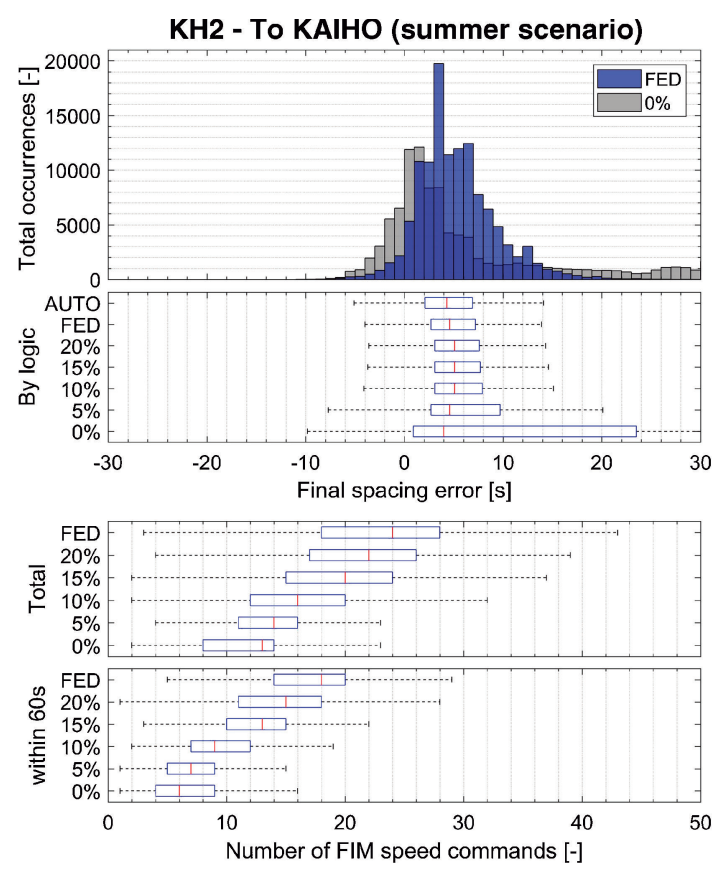

Fig. 7. Results for the en-route scenario to KAIHO (summer data).

Table 5. Accompanying table for KAIHO (summer data).

\begin{tabular}{lccccccc}
\hline \multicolumn{1}{c}{ Logic } & AUTO & FED & $20 \%$ & $15 \%$ & $10 \%$ & $5 \%$ & $0 \%$ \\
\hline Median error [s] & 4.3 & 4.6 & 5.1 & 5.1 & 5.1 & 4.6 & 4.0 \\
Avg. error [s] & 4.78 & 5.22 & 5.58 & 5.67 & 5.89 & 7.92 & 12.93 \\
Std. dev. [s] & 3.67 & 3.87 & 3.99 & 4.06 & 4.46 & 8.59 & 17.05 \\
Mag. (median) [kt] & - & 28 & 28 & 30 & 40 & 50 & 60 \\
Rev. (quartiles) [-] & - & $3-1$ & $3-1$ & $3-1$ & $\mathbf{2 - 1}$ & $2-\mathbf{1}$ & $2-\mathbf{1}$ \\
\hline
\end{tabular}

performance values for RWY22 are found at $[-1.8 \mathrm{~s},-1.0 \mathrm{~s}$, and $0.5 \mathrm{~s}]$ for AUTO, $[-2.6 \mathrm{~s},-1.9 \mathrm{~s}$, and $-0.1 \mathrm{~s}]$ for FED, and $[-0.1 \mathrm{~s}, 6.9 \mathrm{~s}$, and $33.1 \mathrm{~s}]$ for $0 \%$. The median number of speed commands ranges from 20 to 8 . RWY23 yields comparable results, i.e., $[-2.0 \mathrm{~s},-1.2 \mathrm{~s}$, and $0.4 \mathrm{~s}]$ for AUTO, $[-2.9 \mathrm{~s},-2.2 \mathrm{~s}$, and $-0.2 \mathrm{~s}]$ for FED, and $[-0.7 \mathrm{~s}, 7.4 \mathrm{~s}$, and $36.8 \mathrm{~s}$ ] for $0 \%$, with the median number of speed commands ranging from 20 to 10 . The median number of reversals for both scenarios is 1 . For RWY22, the final spacing error standard deviation remains under $5 \mathrm{~s}$ up to $10 \%$ tolerance, while for RWY23, it is just above $5 \mathrm{~s}$ at $10 \%$ tolerance. In both cases, a tolerance of $15 \%$ yields a deviation of less than $3 \mathrm{~s}$.

The larger number of speed commands for the RWY22/23 scenarios compared to the RWY34L/34R scenarios can be attributed to the greater route length and larger number of turns and speed constraints, especially as the approach from ADDUM to RWY34L represents a straight-in approach, while the approaches to RWY22/23 are LDA approaches with sharper turns.

\section{Discussion}

\subsection{Significance of median, mean, standard deviation}

In the previous section, special consideration was given to the median, mean, and standard deviation. As the mean value 

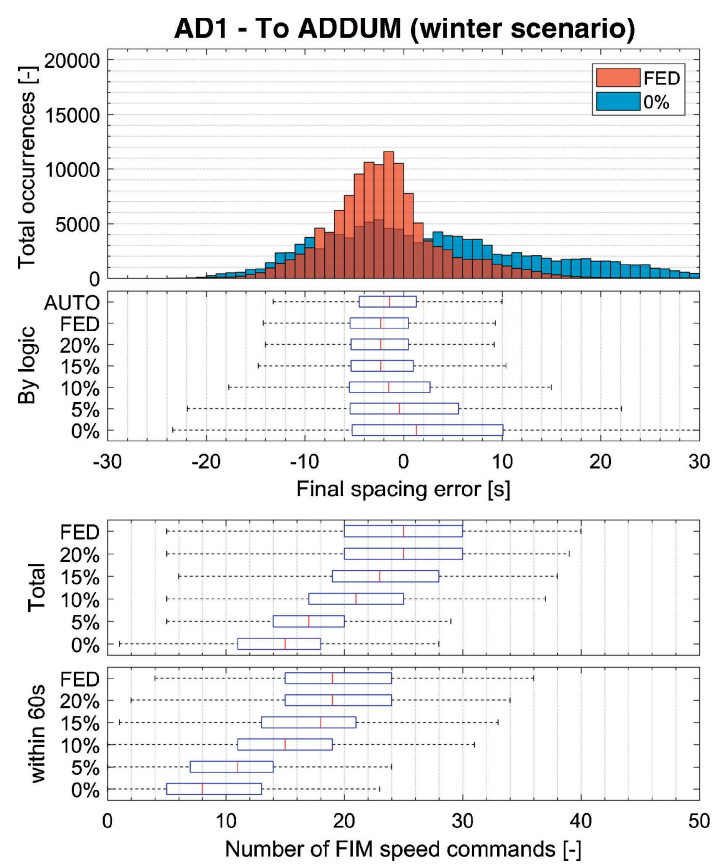

Fig. 8. Results for the en-route scenario to ADDUM (winter data).

Table 6. Accompanying table for ADDUM (winter data).

\begin{tabular}{lccccccc}
\hline \multicolumn{1}{c}{ Logic } & AUTO & FED & $20 \%$ & $15 \%$ & $10 \%$ & $5 \%$ & $0 \%$ \\
\hline Median error [s] & -1.4 & -2.3 & -2.3 & -2.3 & -1.5 & -0.4 & 1.3 \\
Avg. error [s] & -1.24 & -1.96 & -1.80 & -1.36 & -0.48 & 0.89 & 3.07 \\
Std. dev. [s] & 5.50 & 5.80 & 6.02 & 6.66 & 7.55 & 8.85 & 11.23 \\
Mag. (median) [kt] & - & 32 & 32 & 32 & 32 & 35 & 40 \\
Rev. (quartiles) [-] & - & $3-1$ & $3-1$ & $3-1$ & $3-1$ & $3-1$ & $3-1$ \\
\hline
\end{tabular}

is strongly affected by outliers, the median value is better suited for providing an idea of the system's ability to meet the required spacing goal.

As seen in some cases, the median error can reach values of several seconds, indicating that the spacing goal is not met; however, if this difference is systematic and constant, an offset value can be introduced to the nominal spacing goal to compensate for the difference. Furthermore, although the standard deviation is influenced by outliers, it indicates the robustness (reproducibility) of the results. Hence, it has greater importance and the performance requirement is based on it.

\subsection{Comparison of en-route and arrival performance}

The results presented in Section 4 indicate that the arrival scenarios perform significantly better than the en-route scenarios with regard to the standard deviation. Among the en-route scenarios, the KAIHO entry shows the best performance. As the KAIHO entry ends at the lowest altitude with the shortest distance remaining to the runway, it can be regarded as a hybrid scenario of the two scenario types.

Although not all en-route scenarios meet the standard deviation goal of less than $5 \mathrm{~s}$ (and would be regarded as failure), we can assume that the continuation into the arrival scenarios (from ADDUM or STONE) will still allow for successful FIM performance.

These results suggest that the FIM performance improves
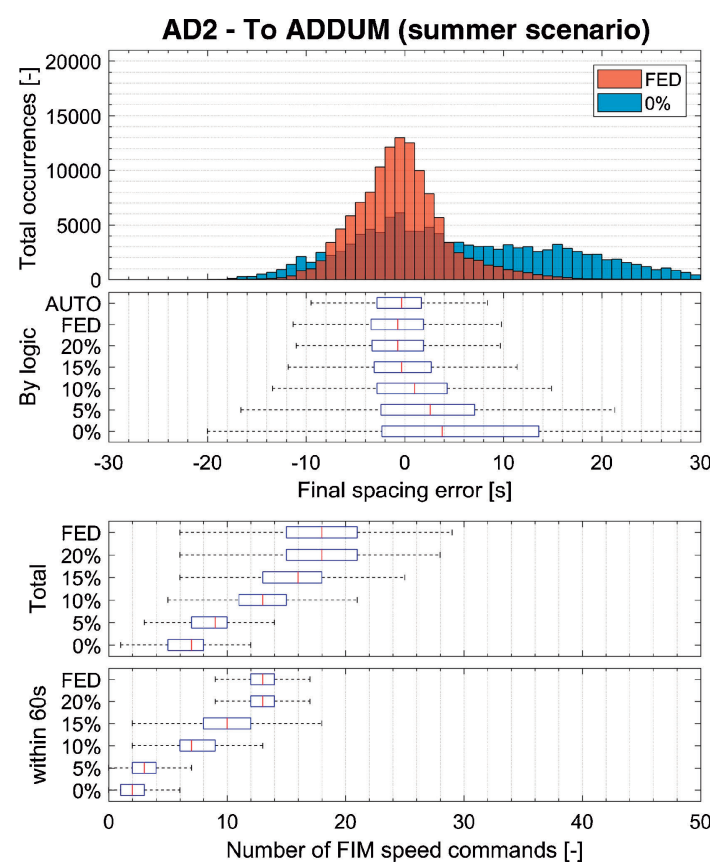

Fig. 9. Results for the en-route scenario to ADDUM (summer data).

Table 7. Accompanying table for ADDUM (summer data).

\begin{tabular}{lccccccc}
\hline \multicolumn{1}{c}{ Logic } & AUTO & FED & $20 \%$ & $15 \%$ & $10 \%$ & $5 \%$ & $0 \%$ \\
\hline Median error [s] & -0.3 & -0.7 & -0.7 & -0.3 & 1.0 & 2.6 & 3.8 \\
Avg. error [s] & -0.17 & -0.46 & -0.32 & 0.32 & 1.36 & 2.98 & 5.65 \\
Std. dev. [s] & 4.46 & 4.73 & 4.87 & 5.36 & 6.10 & 7.54 & 10.67 \\
Mag. (median) [kt] & - & 31 & 31 & 31 & 34 & 35 & 40 \\
Rev. (quartiles) [-] & - & $2-\mathbf{1}$ & $\mathbf{1}$ & $\mathbf{1}$ & $\mathbf{1}$ & $\mathbf{1}$ & $\mathbf{1}$ \\
\hline
\end{tabular}

at lower altitudes, i.e., lower wind and ground speeds. According to the knowledge gained from previous simulations, for the current ASTAR TBO, continuing FIM operation for as long as possible (i.e., all the way in to the low-speed segments) is strongly recommended, and further research effort should be devoted toward performance improvement in high-speed segments.

\subsection{Wind impact}

As seen in the histogram and boxplots for scenario ST1 (Fig. 10), the distribution for the final spacing error extends toward the positive side (delayed arrival). Figure 16 shows the spacing performance separated by the initial waypoint, indicating that delayed arrival is caused by traffic originating from ESCAL. Upon further inspection of the course (CRS) of the routing (see Fig. 2) and the prevailing winds (based on the wind direction $\left(\mathrm{W}_{\mathrm{D}}\right)$ and velocity $\left(\mathrm{W}_{\mathrm{v}}\right)$ listed in Table 14), a significant difference of $138.7 \mathrm{kt}$ in the ground speed (GS) is found in the winter scenario, which results in early arrival for traffic from Y301 and late arrival for traffic from ESCAL.

As ASTAR-based FIM does not include a planned cruise Mach, descend Mach, or descend CAS for the TTF available before a constrained waypoint ${ }^{13)}$ the ground speed and CAS for the TTF cannot be distinguished. This shortcoming adversely affects the quality of FIM speed commands. 

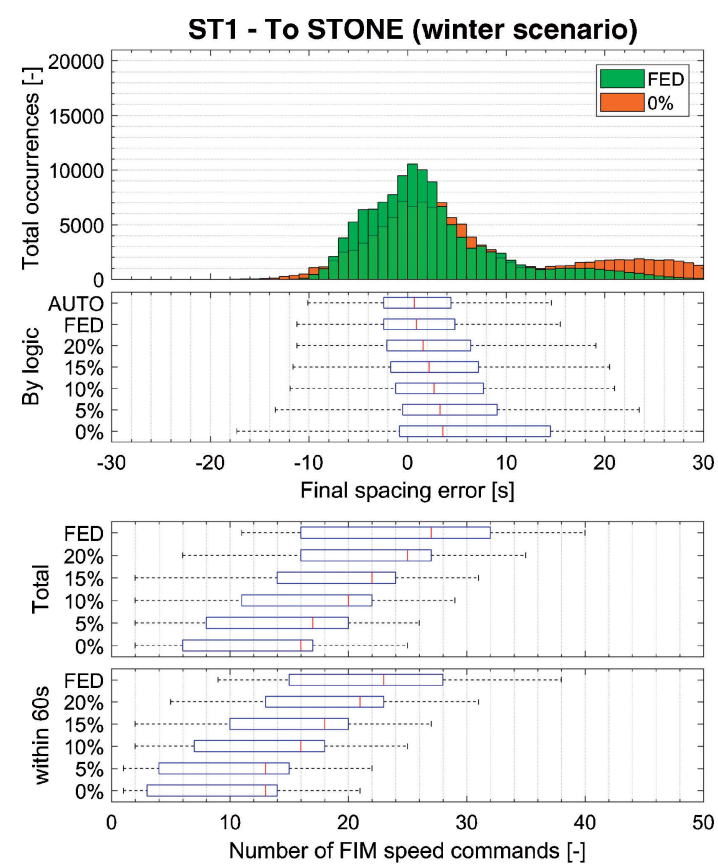

Fig. 10. Results for the en-route scenario to STONE (winter data).

Table 8. Accompanying table for STONE (winter data).

\begin{tabular}{lccccccc}
\hline \multicolumn{1}{c}{ Logic } & AUTO & FED & $20 \%$ & $15 \%$ & $10 \%$ & $5 \%$ & $0 \%$ \\
\hline Median error [s] & 0.7 & 0.9 & 1.6 & 2.2 & 2.7 & 3.3 & 3.6 \\
Avg. error [s] & 1.95 & 2.26 & 3.05 & 3.51 & 3.93 & 5.18 & 7.16 \\
Std. dev. [s] & 6.19 & 6.95 & 7.37 & 7.28 & 7.28 & 8.23 & 11.32 \\
Mag. (median) [kt] & - & 31 & 35 & 35 & 38 & 50 & 60 \\
Rev. (quartiles) [-] & - & $\mathbf{1}$ & $\mathbf{1}$ & $\mathbf{1}$ & $\mathbf{1}$ & $\mathbf{1}$ & $\mathbf{1}$ \\
\hline
\end{tabular}

In addition, when the ground speed difference becomes greater than the $15 \%$ nominal CAS speed range that ASTAR13 can command, late arrival becomes inevitable, causing FIM to fail. This effect becomes more severe at higher wind speeds and a larger angle between opposing traffic. It also contributes to the results of the ADDUM winter scenario. Here, although the wind speeds exceed $120 \mathrm{kt}$ owing to the smaller course difference between traffic arriving from FLUTE and SOPHY (approx. $40^{\circ}$ ), the ground speed difference is only $28 \mathrm{kt}$.

It can be concluded that this phenomenon will be especially prominent in areas affected by dominant winds such as westerlies and jet streams that are prevalent in Japan, and it will make FIM operation more challenging and spacing goals more difficult to achieve. Although FIM is a ground-independent system, ATCOs can play a vital role in achieving successful FIM operation. In particular, in non-benign conditions, the ATCO's understanding of FIM limitations and supervision of current wind conditions are useful for determining and assigning aircraft couplings that are conducive to successful FIM operation and re-arranging those that are not.

\subsection{End-speed tolerance influence}

Figure 17 shows the effect of the end-speed tolerance value on the spacing error standard deviation and the average
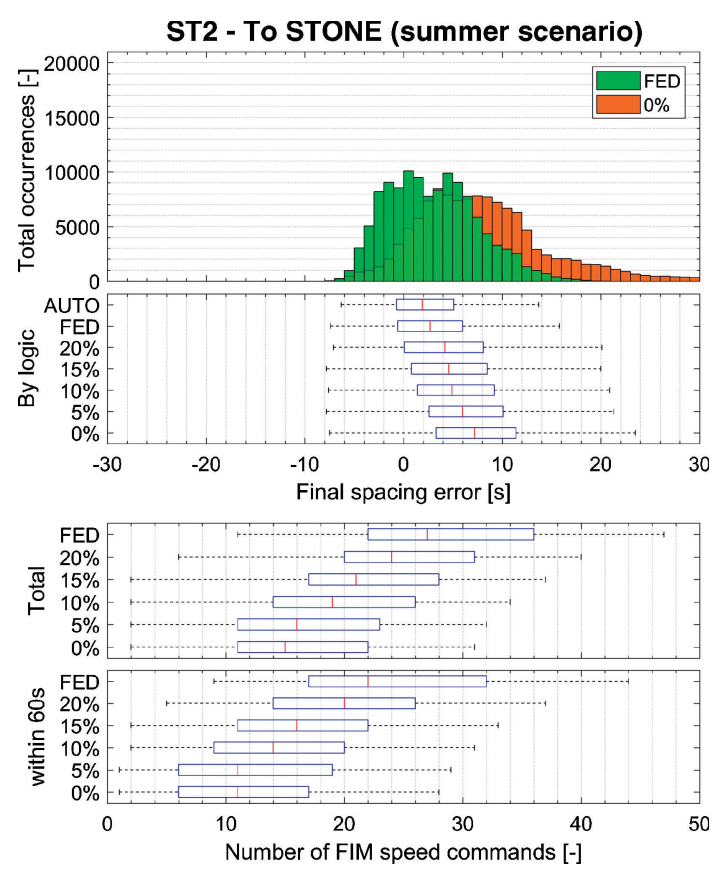

Fig. 11. Results for the en-route scenario to STONE (summer data).

Table 9. Accompanying table for STONE (summer data).

\begin{tabular}{lccccccc}
\hline \multicolumn{1}{c}{ Logic } & AUTO & FED & $20 \%$ & $15 \%$ & $10 \%$ & $5 \%$ & $0 \%$ \\
\hline Median error [s] & 1.9 & 2.7 & 4.2 & 4.6 & 4.9 & 6.0 & 7.2 \\
Avg. error [s] & 2.36 & 2.95 & 4.39 & 4.94 & 5.43 & 6.58 & 8.01 \\
Std. dev. [s] & 3.88 & 4.53 & 5.30 & 5.22 & 5.27 & 5.33 & 6.68 \\
Mag. (median) [kt] & - & 14 & 20 & 31 & 40 & 55 & 65 \\
Rev. (quartiles) [-] & - & $\mathbf{1}$ & $\mathbf{1}$ & $\mathbf{1}$ & $\mathbf{1}$ & $\mathbf{1}$ & $\mathbf{1}$ \\
\hline
\end{tabular}

number of speed commands with the arrival scenarios toward RWY22 (solid line) and RWY23 (dashed line) as an example. The graphs were fitted using cubic interpolation. As the standard deviation and speed commands are of different dimensions, only a relative comparison (i.e., range from the highest to the lowest value) can be made.

In this example, the number of speed commands remains constant until the $20 \%$ tolerance mark, after which a continuous reduction can be observed until the value reaches its minimum at the $0 \%$ mark. Conversely, the standard deviation does not change significantly until the $15 \%$ mark, from which it continues to rise to its maximum value at $0 \%$. The crossing section can be found at a tolerance value of approximately $7 \%$. All of the other scenarios also show the crossing in the range of 5-10\%. However, as indicated by the gray horizontal line, at a tolerance value below $10 \%$ for RWY23 and approximately $9 \%$ for RWY22, the standard deviation exceeds the desired value of $5 \mathrm{~s}$.

Another factor that discourages the use of tolerance values below $10 \%$ is the speed step magnitude, the median value of which reached up to $40 \mathrm{kt}$ for the en-route scenarios, implying that any tolerance lower than $10 \%$ contributes toward greater magnitudes.

Given the requirement of keeping the standard deviation below $5 \mathrm{~s}$ and the fact that the number of commands de- 

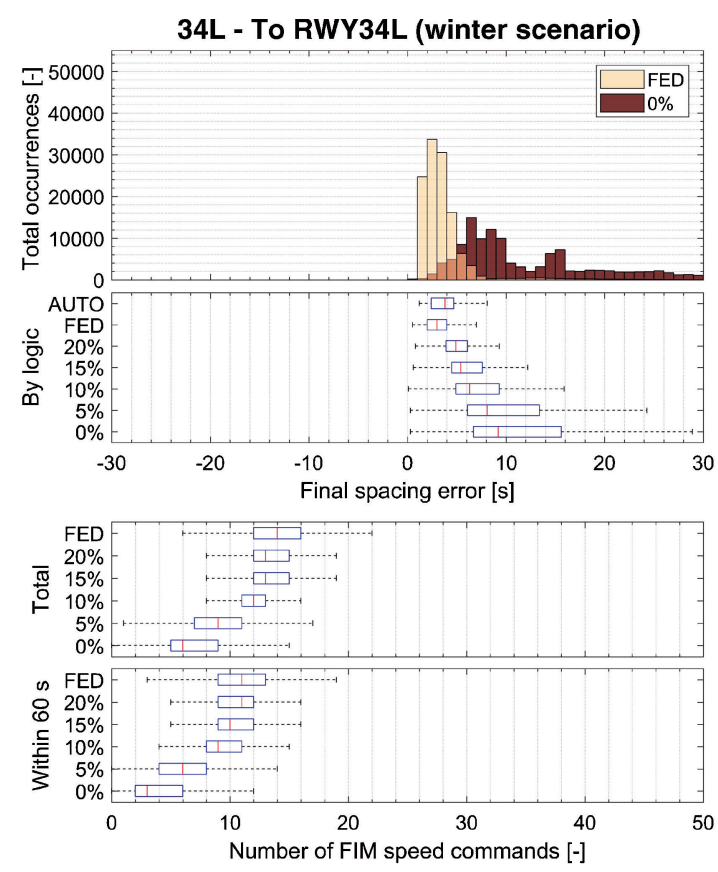

Fig. 12. Results for the arrival scenario to runway 34L (winter data).

Table 10. Accompanying table for runway 34L (winter data).

\begin{tabular}{lccccccc}
\hline \multicolumn{1}{c}{ Logic } & AUTO & FED & $20 \%$ & $15 \%$ & $10 \%$ & $5 \%$ & $0 \%$ \\
\hline Median error [s] & 3.8 & 3.0 & 4.9 & 5.4 & 6.3 & 8.1 & 9.2 \\
Avg. error [s] & 4.04 & 3.52 & 5.37 & 6.13 & 7.02 & 9.41 & 12.19 \\
Std. dev. [s] & 2.63 & 2.66 & 2.62 & 2.80 & 3.21 & 4.43 & 7.35 \\
Mag. (median) [kt] & - & 5 & 5 & 5 & 5 & 15 & 20 \\
Rev. (quartiles) [-] & - & $2-\mathbf{1}$ & $2-\mathbf{1}$ & $2-\mathbf{1}$ & $\mathbf{1}$ & $\mathbf{1 - 0}$ & $\mathbf{1 - 0}$ \\
\hline
\end{tabular}

creases below $20 \%$ tolerance while the standard deviation is not significantly degraded below $15 \%$ tolerance, a tolerance between $10 \%$ and $15 \%$ is found to achieve the best tradeoff between spacing performance and the number of speed commands.

Accordingly, for successful FIM implementation, regulatory prerequisites should be established to allow aircraft engaged in FIM operation to fly at speeds up to $15 \%$ faster than permitted by current constraints. This is the only way in which full use of the FIM and its end-speed tolerance can be guaranteed, thereby reducing the burden on the pilots.

\subsection{Reversals}

For all of the scenarios, the median number of reversals was 1 or greater, implying that one reversal occurred in at least $50 \%$ of all the simulations. ASTAR's tendency for reversals can be attributed to its feed-forward control logic characteristic, which is dependent on the nominal speed. In other words, once the spacing error is reduced, the system will gradually return to the nominal speed. In the case of previous deceleration, this can easily cause a deceleration/ acceleration/deceleration sequence before a planned deceleration.

\subsection{Number of speed commands}

In all of the scenarios, a large number of FIM speed command changes were observed, with most of them being is-
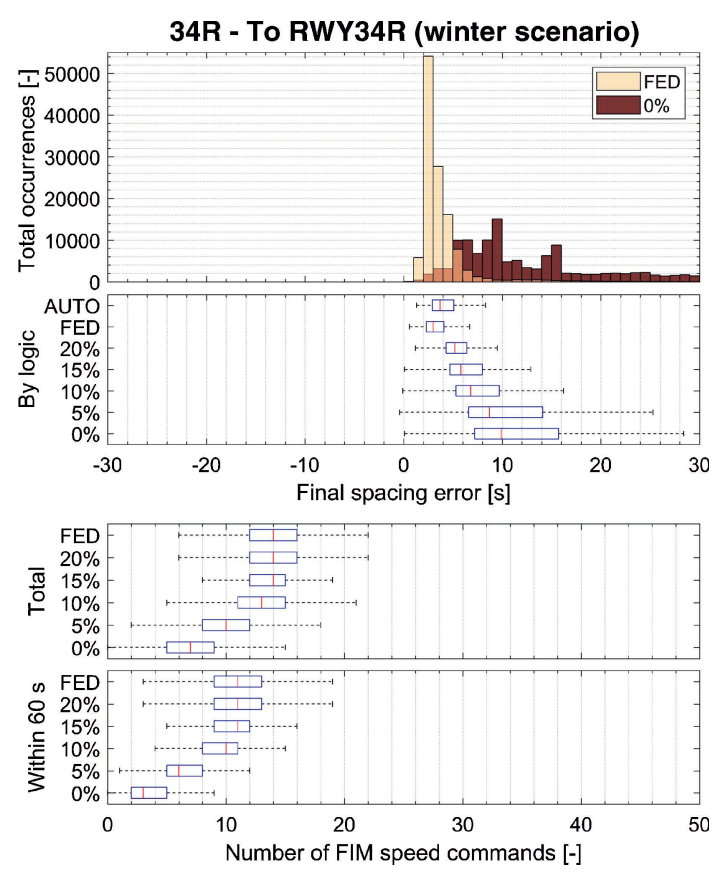

Fig. 13. Results for the arrival scenario to runway 34R (winter data).

Table 11. Accompanying table for runway 34R (winter data).

\begin{tabular}{lccccccc}
\hline \multicolumn{1}{c}{ Logic } & AUTO & FED & $20 \%$ & $15 \%$ & $10 \%$ & $5 \%$ & $0 \%$ \\
\hline Median error [s] & 3.7 & 3.0 & 5.2 & 5.8 & 6.8 & 8.7 & 9.9 \\
Avg. error [s] & 4.33 & 3.65 & 5.59 & 6.39 & 7.41 & 9.96 & 12.64 \\
Std. dev. [s] & 2.48 & 2.50 & 2.51 & 2.79 & 3.23 & 4.51 & 7.30 \\
Mag. (median) [kt] & - & 5 & 5 & 5 & 10 & 15 & 20 \\
Rev. (quartiles) [-] & - & $2-\mathbf{1}$ & $\mathbf{1}$ & $\mathbf{1}-0$ & $\mathbf{1}-0$ & $\mathbf{1}-0$ & $\mathbf{1}-0$ \\
\hline
\end{tabular}

sued less than $60 \mathrm{~s}$ since the last command. This highly undesirable behavior was also highlighted by pilots after the flight test, and a limit of one command per minute was suggested. One reason for the large number of commands is the uniform speed step size of $5 \mathrm{kt}$ used in this simulation. Although a larger step size, as suggested for the CAS cruise phase, ${ }^{13)}$ can reduce the total number of commands, the pilot's comments were based on intervals of $10 \mathrm{kt}$; therefore, the above-mentioned reason is not the sole reason for this issue. Another contributing factor is the feed-forward characteristic of ASTAR. It is presumed that some comments, especially desired rules, as mentioned during the flight test, as well as workload-indicating parameters are difficult to implement in the existing control block; especially, considering its original fully automated background. Therefore, we propose further investigations of not only methods for reducing the number of speed commands, such as the end-speed logic, but also new control concepts that can address the pilot's workload and simplify human-centered operational rules.

\section{Conclusion}

We simulated and analyzed arriving traffic engaged in trajectory-based FIM operation on the most common arrival routes toward Tokyo International Airport. The results showed 

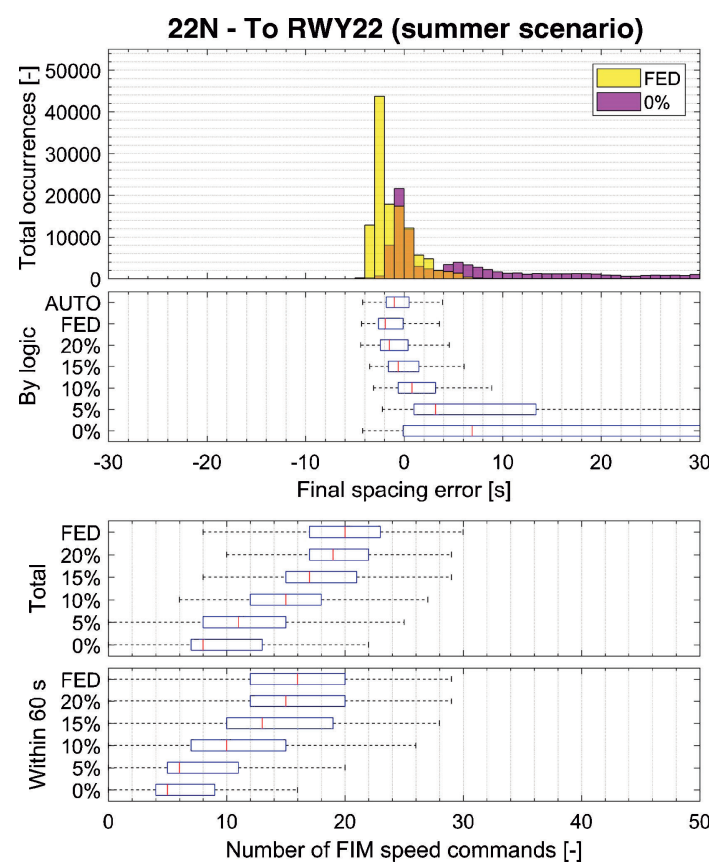

Fig. 14. Results for the arrival scenario to runway 22 (summer data).

Table 12. Accompanying table for runway 22 (summer data).

\begin{tabular}{lccccccc}
\hline \multicolumn{1}{c}{ Logic } & AUTO & FED & $20 \%$ & $15 \%$ & $10 \%$ & $5 \%$ & $0 \%$ \\
\hline Median error [s] & -1.0 & -1.9 & -1.5 & -0.6 & 0.8 & 3.2 & 6.9 \\
Avg. error [s] & -0.54 & -1.18 & -0.81 & 0.00 & 1.93 & 8.92 & 16.61 \\
Std. dev. [s] & 1.89 & 2.00 & 2.25 & 2.26 & 3.87 & 10.94 & 19.69 \\
Mag. (median) [kt] & - & 24 & 24 & 25 & 30 & 35 & 35 \\
Rev. (quartiles) [-] & - & $2-\mathbf{1}$ & $2-\mathbf{1}$ & $2-\mathbf{1}$ & $2-\mathbf{1}$ & $2-\mathbf{1}$ & $\mathbf{1}$ \\
\hline
\end{tabular}

that, especially under low wind conditions (e.g., low flight altitude) as in the case of arrival scenarios, spacing times with standard deviations of $<5 \mathrm{~s}$ can be realized. Furthermore, data showed that an end-speed logic can reduce the total number of speed commands while maintaining adequate spacing performance if a tolerance of $10-15 \%$ is chosen.

However, issues regarding operational feasibility as reported by pilots after the flight test, including the number and frequency of speed commands, were observed. The reasons for such undesired phenomena were discussed, highlighting the need for further investigation of potential solutions.

Accordingly, future research efforts will be devoted toward investigating and proposing alternative control logics for FIM, with the aim of reducing the required pilot interaction and workload as well as avoiding reversals and other undesired behavior.

\section{Acknowledgments}

This research was supported by the Ministry of Education, Culture, Sports, Science and Technology (MEXT) under the "Post-K Computer Exploratory Challenge" (Exploratory Challenge 2: Construction of Models for Interaction among Multiple Socioeconomic Phenomena, Model Development and its Applications for Enabling Robust and Optimized Social Transportation Systems). (Project ID: hp180188)
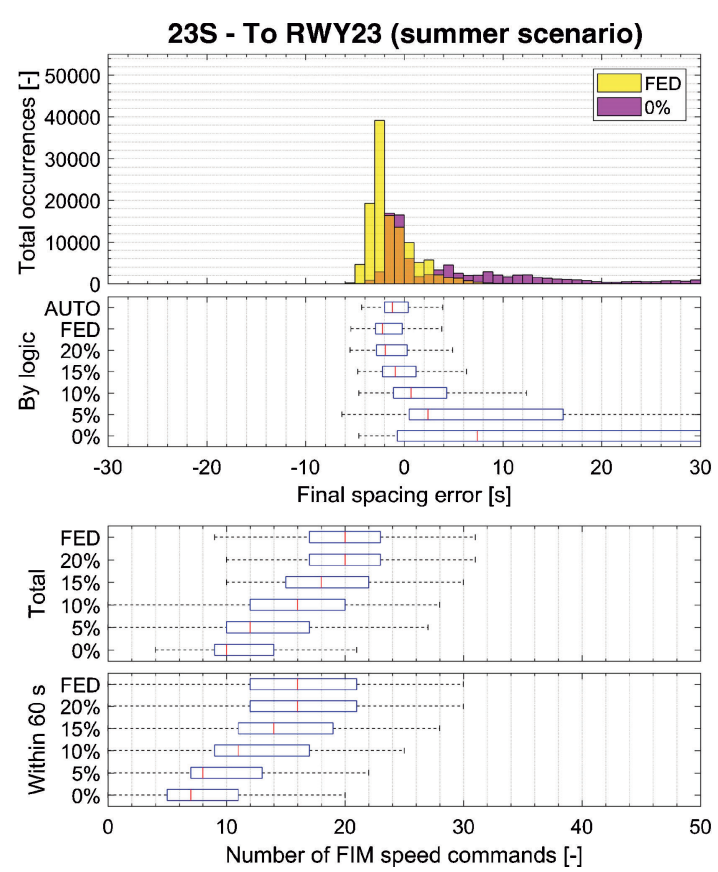

Fig. 15. Results for the arrival scenario to runway 23 (summer data).

Table 13. Accompanying table for runway 23 (summer data).

\begin{tabular}{lccccccc}
\hline \multicolumn{1}{c}{ Logic } & AUTO & FED & $20 \%$ & $15 \%$ & $10 \%$ & $5 \%$ & $0 \%$ \\
\hline Median error [s] & -1.2 & -2.2 & -1.9 & -0.9 & 0.7 & 2.4 & 7.4 \\
Avg. error [s] & -0.54 & -1.36 & -1.07 & -0.15 & 2.34 & 10.26 & 18.03 \\
Std. dev. [s] & 2.29 & 2.41 & 2.57 & 2.89 & 5.06 & 13.85 & 22.30 \\
Mag. (median) [kt] & - & 24 & 24 & 29 & 30 & 30 & 35 \\
Rev. (quartiles) [-] & - & $2-\mathbf{1}$ & $2-\mathbf{1}$ & $2-\mathbf{1}$ & $2-\mathbf{1}$ & $2-\mathbf{1}$ & $\mathbf{1}$ \\
\hline
\end{tabular}

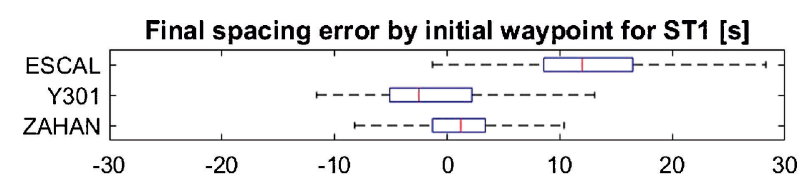

Fig. 16. Results of ST1, separated by the initial waypoint.

Table 14. Wind effects on ground speed for ST1.

\begin{tabular}{cccclc}
\hline Initial WP & ${\text { CRS }\left[{ }^{\circ}\right]}$ & $\mathrm{W}_{\mathrm{D}}\left[{ }^{\circ}\right]$ & $\mathrm{W}_{\mathrm{v}}[\mathrm{kt}]$ & Wind type & GS [kt] \\
\hline ESCAL & 247.2 & 269.5 & 89.9 & Headwind & 387.8 \\
Y301 & 140.0 & 278.8 & 75.3 & Qrt. Tailwind & 526.1 \\
\hline
\end{tabular}

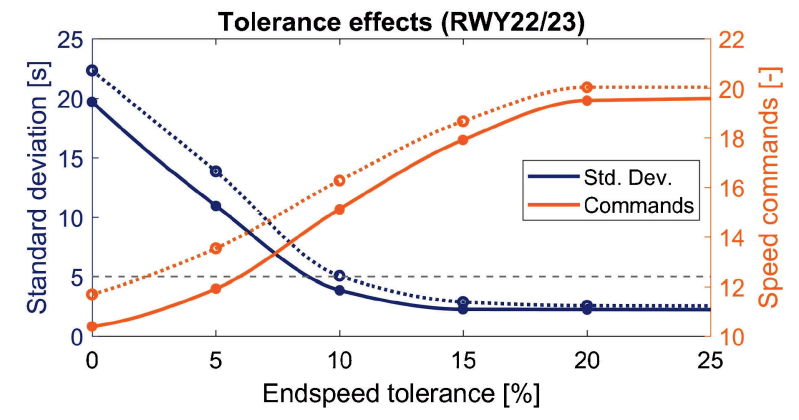

Fig. 17. Effect of end-speed tolerance on average number of speed commands and standard deviation with RWY22 (solid line) and RWY23 (dashed line) as an example. 


\section{References}

1) ICAO: 2016-2030 Global Air Navigation Plan, Doc 9750, 2015.

2) Study Group for the Future Air Traffic Systems: Long-term Vision for the Future Air Traffic Systems CARATS, Collaborative Actions for Renovations of Air Traffic Systems, https://www.mlit.go.jp/common/ 000128185.pdf (accessed August 24th, 2017).

3) Itoh, E. and Uejima, K.: Applying Flight-deck Interval Management Based Continuous Descent Operation for Arrival Air Traffic to Tokyo International Airport, 10th ATM Seminar, Chicago, USA, 2013.

4) Itoh, E., Uejima, K., Kakichi, Y., and Suzuki, S.: Modeling and Simulation Study on Airborne-based Energy Saving Arrivals to Tokyo International Airport, AIAA Guidance, Navigation, and Control (GNC) Conference, Guidance, Navigation, and Control and Co-located Conferences, AIAA 2013-4779, 2013.

5) Riedel, T., Itoh, E., and Takahashi, M.: Investigating Aircraft Speed Control Logics for Interval Management Targeting Arrival Traffic to Tokyo International Airport, Asia-Pacific International Symposium on Aerospace Technology 2017, Seoul, South Korea, 2017.

6) Riedel, T., Itoh, E., Tatsukawa, T., and Takahashi, M.: Preliminary Study on Interval Management for Improving Aircraft Speed Command Behavior, 55th JSASS Aircraft Symposium, Matsue-shi, Japan, 2017.

7) Swieringa, K. A., Wilson, S. R., Baxley, B. T., Roper, R. D., Abbott, T. S., Levitt, I., and Scharl, J.: Flight Test Evaluation of the ATD-1 Interval Management Application, 17th AIAA Aviation Technology, Integration, and Operations Conference, AIAA Aviation Forum, AIAA 2017-4094, 2017.

8) Baxley, B. T., Swieringa, K. A., Wilson, S. R., Roper, R. D., Hubbs, C., Goess, P., and Shay, R.: Flight Crew Survey Responses from the Interval Management (IM) Avionics Phase 2 Flight Test, 17th AIAA Aviation Technology, Integration, and Operations Conference, AIAA Aviation Forum, AIAA 2017-4095, 2017.

9) Baxley, B. T., Swieringa, K. A., Roper, R. D., Hubbs, C., Goess, P., and Shay, R.: Recommended Changes to Interval Management to Achieve Operational Implementation, 2017 IEEE/AIAA 36th Digital Avionics Systems Conference (DASC), St. Petersburg, FL, 2017.
10) Weitz, L. and Swieringa, K. A.: Comparing Interval Management Control Laws for Steady-State Errors and String Stability, 2018 AIAA Guidance, Navigation, and Control Conference, AIAA SciTech Forum, Kissimmee, FL, USA, 2018.

11) Miyazaki, H., Kusano, Y., Shinjou, N., Shoji, F., Yokokawa, M., and Watanabe, T.: Overview of the K Computer System, Fujitsu Sci. Tech. J., 48 (2012), pp. 255-264.

12) RTCA SC-186. Minimal Operational Performance Standards (MOPS) for Flight-deck Interval Management (FIM), RTCA DO-361, 2015.

13) Abbot, T. S.: An Overview of a Trajectory-based Solution for En Route and Terminal Area Self-spacing: Seventh Revision, NASA/ CR-2015-218794, 2015.

14) Abbot, T. S.: A Trajectory Algorithm to Support En Route and Terminal Area Self-spacing Concepts: Third Revision, NASA/CR-2014218288, 2014.

15) Abbot, T. S.: An Overview of a Trajectory-based Solution for En Route and Terminal Area Self-spacing to Include Parallel Runway Operations, NASA/CR-2011-217194, 2011.

16) Baxley, B. T., Johnson, W. C., Swenson, H. N., Robinson, J. E., Prevot, T., Callantine, T. J., Scardina, J., and Greene, M.: Air Traffic Management Technology Demonstration-1 Concept of Operations (ATD-1 ConOps) Version 2.0, NASA TM-2013- 218040, 2013.

17) Japan Civil Aviation Bureau: Aeronautical Information Publication AD2-24, RJTT Charts Related to an Aerodrome, Effective, 19th July 2018.

18) Itoh, E., Fukushima, S., Hirabayashi, H., Wickramasinghe, N., and Toratani, D.: Evaluating Energy-Saving Arrivals of Wide-Body Passenger Aircraft via Flight-Simulator Experiments, J. Aircraft, 55, 6 (2018), pp. 2427-2443, http://arc.aiaa.org/doi/abs/10.2514/1.C034348

19) Itoh, E., Fukushima, S., Hirabayashi, H., and Wickramasinghe, N. K.: Simulation Evaluation on Fixed-flight Path Angle Descent, J. Jpn. Soc. Aeronaut. Space Sci., 64 (2016), pp. 50-57.

Keumjin Lee Associate Editor 